\title{
ARTICLE
}

\section{The study on the interaction of Epigallocatechin gallate with bovine serum Albumin}

Gombosuren Davaadulam*, Maamuu Tamara and Nyamaa Gerelsuren

Institute of Physics and Technology, Mongolian Academy of Sciences, Ulaanbaatar, Mongolia

ARTICLE INFO: Received: 25 Oct, 2019; Accepted: 30 Jan, 2020

\begin{abstract}
In this study, we investigated the interaction of epigallocatechin gallate (EGCG) with bovine serum albumin (BSA) by fluorescence method and protein-ligand docking. We separated EGCG from green tea using the chromatographic method and analyzed structural activity relationships of the EGCG. The results show that EGCG is a strong quencher of BSA fluorescence and binds with BSA with high affinity. The binding parameters (binding constant, the number of binding sites) were determined by the Ward equation. From the thermodynamic parameters, calculated according to the van't Hoff equation, the enthalpy change $\Delta H^{\circ}$, and entropy change $\Delta S^{\circ}$ were found to be $-22.67 \mathrm{~kJ} / \mathrm{mol}$ and $14.92 \mathrm{~J} / \mathrm{mol} / \mathrm{K}$, respectively. These values suggest that apart from an initial hydrophobic association, the complex is held together by electrostatic and hydrogen bonding. In the docking simulation, the lowest free energies for the interaction of EGCG with tryptophan residues was $-21.92 \mathrm{~kJ} / \mathrm{mol}$ (Trp 134) and $-24.7 \mathrm{~kJ} / \mathrm{mol}$ (Trp213). The binding between EGCG and BSA consists of hydrogen bonds (Trp213) and hydrophobic interactions (Trp134).
\end{abstract}

Keywords: Chromatographic method; binding parameter; fluorescence; thermodynamic parameter;

\section{INTRODUCTION}

Green tea contains catechin complex such as (+)-catechin, (-)-epicatechin, (-)-epicatechin gallate, (-)-epigallocatechin, (-)epigallocatechin gallate. From these, EGCG is the richest in green tea, which has a broad range of biological activity including antioxidants [1], antibacterial [2], anti-cancer [3], anti-diabetic [4] and cancer preventive activities [5].
In several in vitro and in vivo studies, EGCG is a compound of biological activity with aromatic, stronger antioxidants compared to other catechins [6]. EGCG's biological activities function related to its structure (Figure 1). The role of EGCG in health can, therefore, be understood only in the context of its binding to serum albumin.

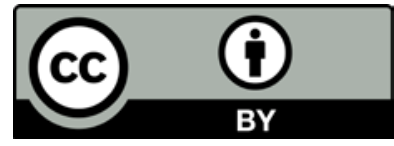

The Author(s). 2018 Open access This article is distributed under the terms of the Creative Commons Attribution 4.0 International License (https://creativecommons.org/licenses/by/4.0/), which permits unrestricted use, distribution, and reproduction in any medium, provided you give appropriate credit to the original author(s) and the source, provide a link to the Creative Commons license, and indicate if changes were made. 


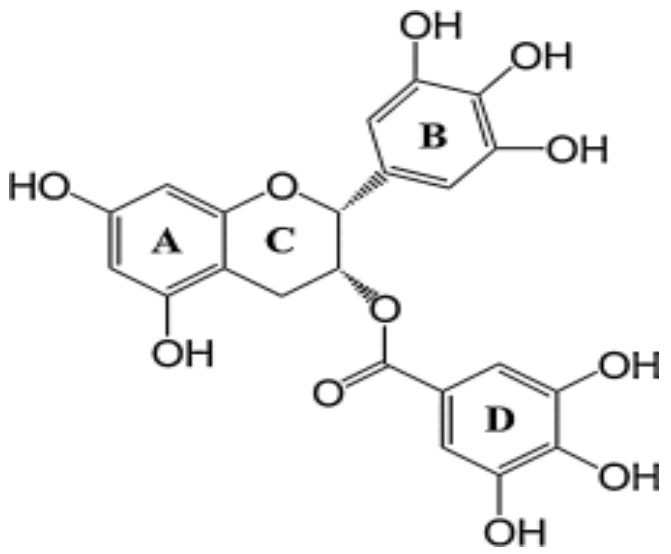

Figure 1. Chemical structure of EGCG

BSA binds and transports many small molecules (drugs, vitamins, fatty acids, hormones, etc.) through the bloodstream [7]. BSA has been one of the most extensively studied of this group of proteins, due to its structural homology with HSA (76\% identity and $88 \%$ similarity) [8]. BSA is a globular protein consisting of 583 amino acid residues with 2 tryptophan residues (Trp134 and Trp213) and has three hydrophobic pockets. Trp134 is in a solvent-exposed region of subdomain IB and is highly accessible, whereas Trp213 is buried in the hydrophobic pocket

\section{MATERIALS AND METHODS}

\section{Samples and reagents}

The Sencha green tea (Camellia sinensis) used as crude extracts were obtained from the Mercury Market (Ulaanbaatar, Mongolia). EGCG standard and other reagents of analytical grade were purchased from Wako Pure Chemical Industries (Osaka, Japan), SigmaAldrich (Germany), and Damao Chemical Reagent Factory (Tianjin, China).

Preparation of green tea extracts and separation of catechins fraction

A mixture of catechins was extracted from the Sencha green tea. The obtained extract was partially separated by column chromatography using chloroform-water (1:1, $\mathrm{v} / \mathrm{v})$ and water-ethyl acetate $(1: 1, \mathrm{v} / \mathrm{v})$ solvent systems [13].

\section{High-performance liquid chromatography (HPLC) analysis}

near the drug binding site in subdomain IIA. Most investigations carried out on the interaction serum albumin with compounds have shown biological activity, which have been reported in the literature [9-12].

The study of interactions between catechins and albumin is important for food chemistry, pharmacology, and clinical medicine. Therefore, in this work, the interaction of BSA with EGCG has been investigated by fluorescence method and protein-ligand docking.

EGCG in the catechins fractions was separated using the Nexera X2 HPLC system equipped with C18-RP-HPLC $250 \times 4.0 \mathrm{~mm} \times 5 \mu \mathrm{m}$. The mobile phase was composed of water/acetonitrile (A solution), acetic acid (B solution) and flow rate was 1 $\mathrm{ml} / \mathrm{min}$.

Fluorescence spectroscopy and quenching constant calculation

Solutions of EGCG and BSA were prepared in a $0.01 \mathrm{M}$ phosphate buffer, $\mathrm{pH} 7.4$ containing $0.15 \mathrm{M} \mathrm{NaCl}$. Fluorescence measurements were performed on a Hitachi Spectrofluorometer Model F-4600 equipped with Xenon Lamp and a slit width of $10 \mathrm{~nm}$, using a $1 \mathrm{~cm}$ quartz cell. These studies were carried out to select optimum protein and EGCG concentrations for the interaction. BSA concentration was kept fixed at $100 \mu \mathrm{M}$ and EGCG concentration was varied from 10 to 50 
$\mu \mathrm{M}$. Fluorescence spectra were recorded at different temperatures in the range of 300-500 $\mathrm{nm}$ upon excitation at $281 \mathrm{~nm}$.
Fluorescence data were analyzed by the following equation. The following has been revealed for equivalent and independent binding sites [14]:

$$
\log \left(F_{0}-F\right) / F_{0}=\log K_{b}+n \log [E G C G]
$$

$F_{0}$ and $F$ are the fluorescence intensities before and after the addition of EGCG, respectively. $K_{b}$ is the binding constant, [EGCG] is the concentration of EGCG, $n$ is the number of binding sites.

$$
F_{0} / F=1+K_{S V}[E G C G]=1+k_{q} \times \tau_{0}[E G C G]
$$

$K_{S V}$ is the quenching constant of Stern-Volmer. $k_{q}$ is the bimolecular quenching rate constant, and $\tau_{0}$ is the lifetime of the fluorophore in the absence of the EGCG $\left(\tau_{0}\right.$ is about $2.89 \times 10^{-9}$ s).

Thermodynamics of BSA-EGCG interactions
Fluorescence quenching is described by Stern-Volmer equation [15]:

$$
\log K_{b}=-\frac{\Delta H^{\circ}}{R T}+\frac{\Delta S^{\circ}}{R}
$$

$K_{b}$ is the binding constant at the corresponding temperature and $R$ is the gas constant. Utilizing
Thermodynamics parameters, such as the free energy change $\left(\Delta \mathrm{G}^{\circ}\right)$, the enthalpy change $\left(\Delta \mathrm{H}^{\circ}\right)$, entropy change $\left(\Delta \mathrm{S}^{\circ}\right)$ for BSA-EGCG interactions were calculated at 3 different temperatures, 29,31 , and $37^{\circ} \mathrm{C}$ by the van't Hoff equations [16]:

$$
\Delta G^{\circ}=-\mathrm{RTLog} K_{b}
$$

Docking studies of BSA-EGCG interactions

Molecular docking is one of the important methods for structure biological and making drug design in silico. In order to understand the interaction of BSA-EGCG at the atomic level, this simulation is studied by molecular docking. The structure of BSA was

\section{RESULTS AND DISCUSSION}

\section{HPLC analysis}

We completely separated EGCG from other compounds in the Sencha green tea
$K_{b}$, the free energy change $(\Delta \mathrm{G})$ value can be estimated using the following equation [16]: taken from Protein Data Bank (PDB ID: 4F5S). The 3D structure of EGCG was taken from (ChEBI: 4806) Chemical Entities of Biological Interest as a MOL file. The mechanism of the BSA-EGCG interaction was performed using AutoDock Tool 4.2 v.1.5.6 [17].

extract using a silica gel column and HPLC chromatographic method. Chromatographic separation of EGCG is shown in Figure 2. 


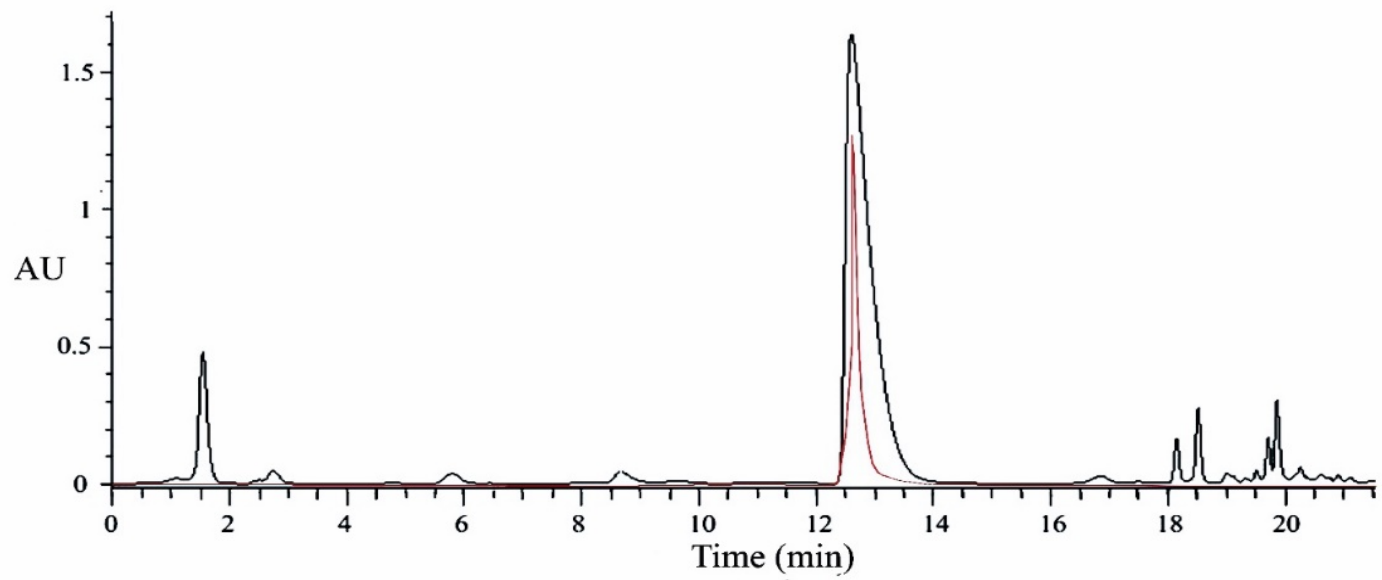

Figure 2. HPLC chromatogram of standard EGCG (red) and the separated EGCG (black). The separation was performed at $25^{\circ} \mathrm{C}$ and the analytical wavelength was set up for $280 \mathrm{~nm}$

The standard peak of EGCG was identified as the position with a retention time $\left(t_{R}\right)$ of 12.435 min (red), while in the separated EGCG was detected at 12.44 min (Figure 2 (black)). In our case, the peak of EGCG has selectivity factor $(\alpha)=1.99$ and the peak area has linear correlation $(\mathrm{r}=0.9994, \mathrm{p}<0.01)$. Finally, we purified $46.8 \pm 0.21 \mathrm{mg} / \mathrm{g}$ of EGCG

\section{Fluorescence quenching and} thermodynamics analysis

Quenching of protein intrinsic fluorescence was employed for a more detailed study of BSA-EGCG interactions. The fluorescence spectra of BSA in the absence and presence of EGCG were measured with an excitation wavelength of $281 \mathrm{~nm}$, which is attributed to tryptophan residues only. The fluorescence intensity of BSA gradually decreased with the rising concentration of EGCG, showing that EGCG binds with the BSA. The fluorescence titration spectra at $25^{\circ} \mathrm{C}$ are shown in Figure 3. A smaller blue shift (from 346 to $339 \mathrm{~nm}$ ) in $\lambda_{e m}^{\max }$ of tryptophan fluorescence was observed in the interaction of EGCG with BSA. The blue shift in the peak of fluorescence emission of EGCG as well as the increased intensity in the presence of protein are indicative of the interactions between EGCG and BSA. Moreover, EGCG was seen to quench the fluorescence of BSA, meaning that its two tryptophan residues were involved in BSA- EGCG interactions.

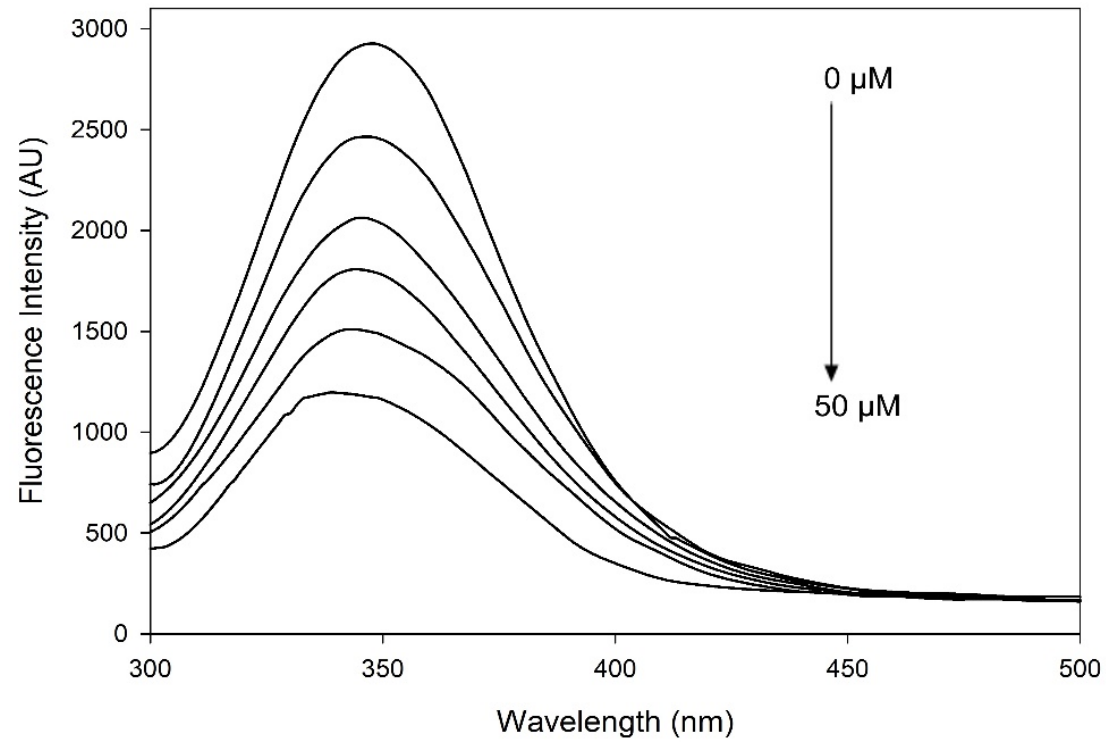

Figure 3. Fluorescence quenching spectra of BSA $(10 \mu \mathrm{M})$ in the presence of different concentrations of EGCG $(10-50 \mu \mathrm{M}) . \mathrm{pH} 7 \cdot 4 ; \mathrm{T}=25^{\circ} \mathrm{C}$ 
From the Stern-Volmer plots for the quenching of intrinsic fluorescence of tryptophan residues shown in Figure 4, the values for quenching constant $K_{S V}$ was calculated as $3.13 \times 10^{4}, 2.28 \times 10^{4}$, and $1.79 \times$ $10^{4} \mathrm{M}$ at 25,31 , and $37^{\circ}$, respectively. It was also found that $K_{S V}$ decreases with an increase in temperature, suggesting that the temperature has a pronounced effect on BSA-EGCG interactions.

The equation (2) was applied to determine the bimolecular quenching rate constants $\left(k_{q}\right)$ by linear regression of plots of
$F_{0} / F$ versus [EGCG] (Figure 4). The quenching rate constants are summarized in Table 1 . The values of $k_{q}$ for BSA-EGCG interaction are higher than the diffusion-limited rate constant of the biomolecule $\left(k_{\text {diff }}=1.0 \mathrm{x}\right.$ $10^{10} \mathrm{l} \mathrm{mol}^{-1} \mathrm{~s}^{-1}$ ) which confirmed that the static quenching mechanism is the key reason for protein fluorescence quenching [18]. Considering that the values of $k_{q}$ were higher than $k_{\text {diff }}$, the static quenching mechanism is most probably the main reason for protein fluorescence quenching by catechins in the range of concentrations $10-50 \mu \mathrm{M}$.

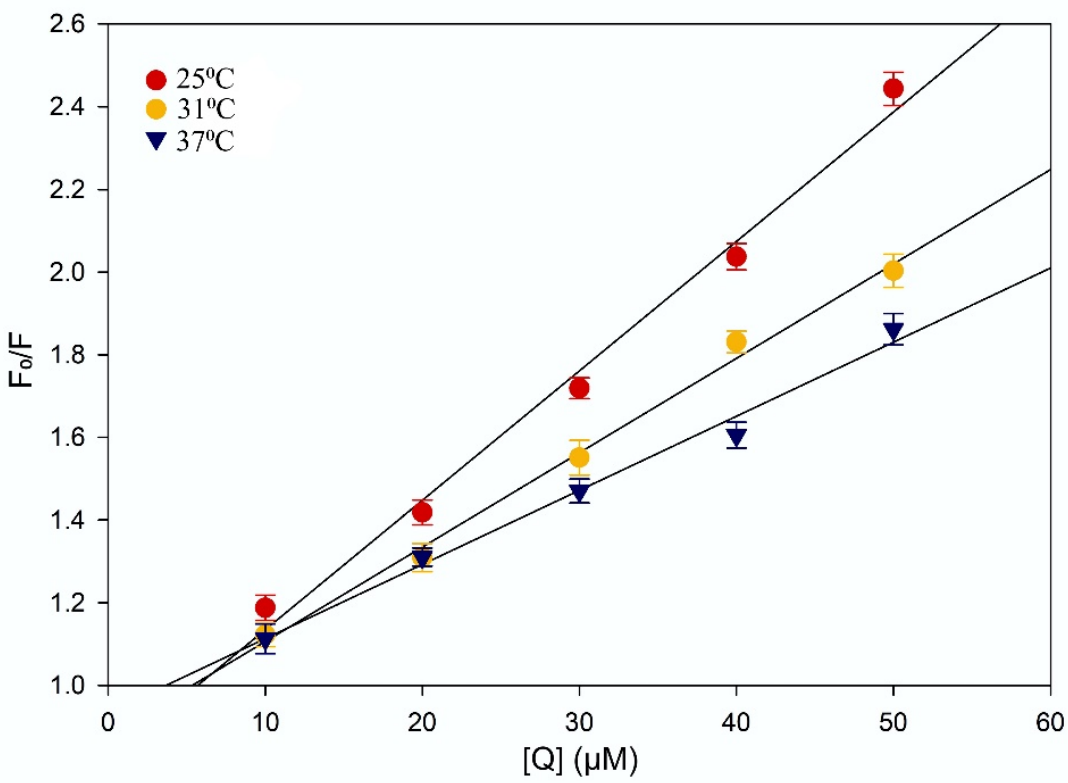

Figure 4. Stern-Volmer plots for the quenching of the fluorescence of the Trp residues of BSA. Correlation coefficients $(R)$ were found in the range of 0.989-0.995

The binding constants $K_{b}$ and binding site $\mathrm{n}$ for BSA-EGCG interactions at 25, 31, and $37^{\circ} \mathrm{C}$ were calculated using Logarithmic plots (Figure 5). The logarithmic plots for determining binding parameters indicate that EGCG binds to a single site on BSA, which is in agreement with the number of binding sites $\mathrm{n}$. The value of the number of binding sites ranged from 0.83 to 1.06. From the Ward equation, the binding constants $K_{b}$ at 25, 31, and $37^{\circ} \mathrm{C}$ were calculated and the values are $5.72 \times 10^{4}, 6.15 \times 10^{4}$, and $6.67 \times 10^{4} \mathrm{M}$, respectively (Table 1 ). 


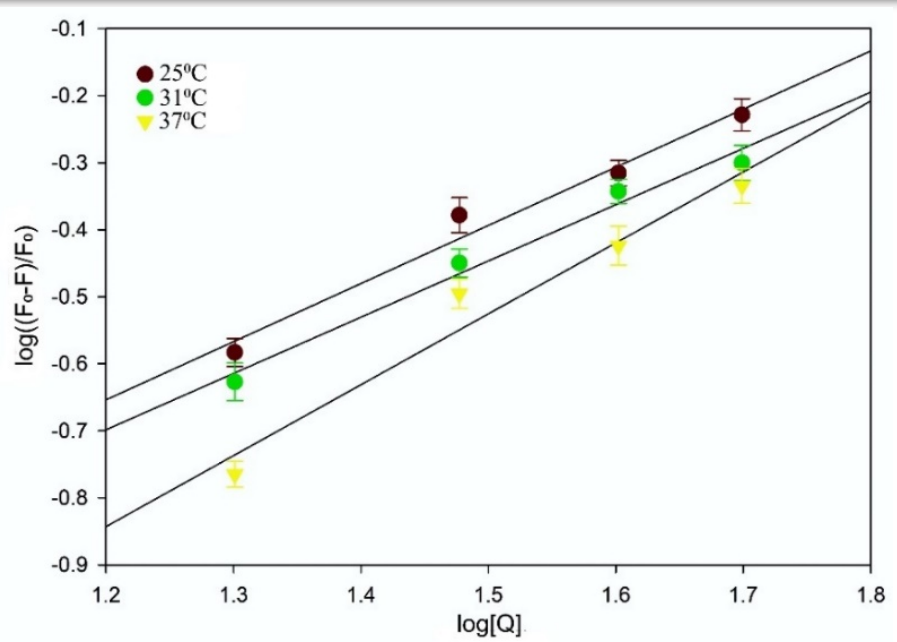

Figure 5. Logarithmic plots of EGCG-HSA interactions. Correlation coefficients $(R)$ were found in the range 0.987-0.992

Hydrogen bonds, van der Waals, hydrophobic, and electrostatic interactions are the basic interactions that have a key role in the protein-ligand binding [19]. The thermodynamic parameters of interaction provide an understanding of binding mode. The thermodynamic parameters involved in the binding process were calculated using van't Hoff equation. The obtained values from this equation are presented in Table 1.

As seen in Table 1, BSA-EGCG interaction is an exothermic process accompanied by the negative values of free energy changes $\left(\Delta \mathrm{G}^{\circ}\right)$ and the positive values of entropy change $(\Delta S)$. Moreover, the negative values of $\Delta \mathrm{G}^{\circ}$ indicate that the binding between BSA and EGCG proceeds spontaneously. For typical hydrophobic interaction, both entropy change $\left(\Delta S^{\circ}\right)$ and enthalpy change $\left(\Delta H^{\circ}\right)$, are positive, which was different in our results such as $\Delta \mathrm{S}^{\circ}=14.92 \mathrm{~J} / \mathrm{mol} / \mathrm{K}$ (positive) and $\Delta \mathrm{H}^{\circ}=$ $22.67 \mathrm{~kJ} / \mathrm{mol}$ (negative). The positive entropy observed accounts for hydrophobic interactions and negative enthalpy may play a role in electrostatic interactions, which is also inferred from the decrease in quenching constant with an increase in ionic strength [20].

Table 1. The bimolecular quenching rate constants (Kq), binding constants (Kb), number of binding sites ( $\mathrm{n})$ and free energy change $\left(\Delta G_{0}\right)$ of EGCG-BSA interactions

\begin{tabular}{|c|c|c|c|c|c|c|}
\hline$T,{ }^{\circ} \mathrm{C}$ & $K_{q}\left(x 10^{12} M^{-1} s^{-1}\right)$ & $K_{b}\left(x 10^{4} M^{-1}\right)$ & $n$ & $\begin{array}{c}\Delta \boldsymbol{G} \\
\left(\mathrm{kJmol}^{-1}\right)\end{array}$ & $\begin{array}{c}\boldsymbol{\Delta} \boldsymbol{H}^{\circ} \\
\left(\mathrm{kJmol}^{-1}\right)\end{array}$ & $\begin{array}{c}\Delta \boldsymbol{S}^{\circ} \\
\left(\mathrm{J} \mathrm{K}^{-1} \mathrm{~mol}^{-1}\right)\end{array}$ \\
\hline 25 & 10.82 & 5.7158 & 0.83 & -27.12 & \multirow{3}{*}{-22.67} & \multirow{3}{*}{14.92} \\
\hline 31 & 7.88 & 6.1521 & 0.97 & -27.86 & & \\
\hline 37 & 6.19 & 6.6659 & 1.06 & -28.59 & & \\
\hline
\end{tabular}

\section{Docking studies of BSA-EGCG interactions}

BSA has two Trp residues, for example Trp134 is located on the surface of the molecule, and Trp213 is located within drugbinding site I [21]. The docking analysis was conducted to evaluate the binding affinity of BSA to EGCG (Figure 6). From the results of simulation, EGCG was observed to interact with both Trp residues. The lowest binding energy for the affinity of EGCG with Trp134 and Trp-213 was $-21.92 \mathrm{~kJ} / \mathrm{mol}$ and -24.7 $\mathrm{kJ} / \mathrm{mol}$ respectively. In the drug binding site, a hydrogen bond was observed between EGCG and Trp213, which may explain why the affinity against Trp-213 was calculated to be stronger than that against Trp-134. EGCG interactions with nearby Trp134 residues are dominated by van der Waals interactions and the hydrophobic effect. 

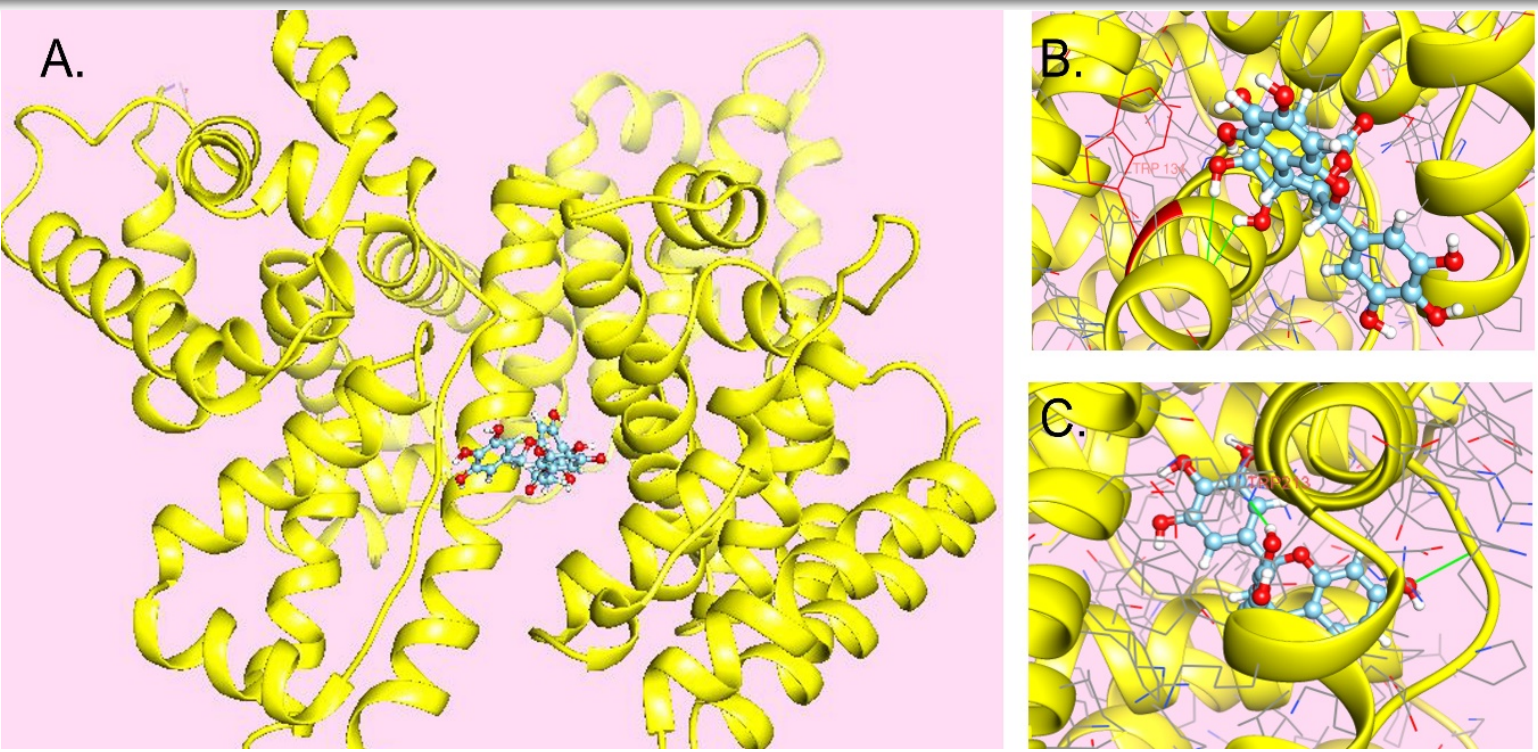

Figure 6. The interaction of EGCG with Trp residues in BSA by the docking simulation. A. complexation between BSA and EGCG in the drug binding site I. B. The docking pose of EGCG (blue ball \& stick) against Trp-134 at the protein surface (B) and against Trp-213 in the drug-binding site I (C). Hydrogen bonds were described as green solid lines. The molecular surface of the protein was colored as a yellow cartoon. The figure was made by the UCSF Chimera [22]

\section{CONCLUSIONS}

In summary, the interactions of EGCG with BSA has been presented by fluorescence methods and docking studies. The results show that EGCG is a strong quencher of the fluorescence of BSA and binds to the BSA with high affinity. The number of binding sites was roughly one for BSA-EGCG interactions. These interaction processes were spontaneous based on free energy changes. The binding forces are shown to be hydrophobic and electrostatic in nature and stabilized with hydrogen bonding. Moreover, in the drugbinding site I, the interaction of EGCG with Trp213 in BSA bound to hydrogen bonding. This report has a significance in the field of pharmacology and food chemistry.

\section{REFERENCES}

1. Rice-Evans, C. Implications of the mechanisms of action of tea polyphenols as antioxidants in vitro for chemoprevention in humans. Proc. Soc. Exp. Biol. Med., (1999) 220, 262-266. https://doi.org/10.1046/j.1525-1373.1999.d01-45.x

2. Mehdi Nikoo, Joe M. Regenstein, and Hassan Ahmadi Gavlighi, Antioxidant and Antimicrobial Activities of (-)-Epigallocatechin-3-gallate (EGCG) and its Potential to Preserve the Quality and Safety of Foods. Comprehensive Reviewsin Food Science and Food Safety (2018) 17, 732-753. https://doi.org/10.1111/1541-4337.12346

3. Isemura M., Saeki K., Kimura T., Hayakawa S., Minami T., Sazuka M. Tea catechins and related polyphenols as anti-cancer agents. Biofactors. (2000)13(1-4), (81-85).

https://doi.org/10.1002/biof.5520130114

4. Saeed Samarghandian, Mohsen Azimi-Nezhad, and Tahereh Farkhondeh, Catechin Treatment Ameliorates Diabetes and Its Complications in Streptozotocin-Induced Diabetic Rats. Dose Response. (2017) 15(1). https://doi.org/10.1177/1559325817691158

5. Yang C., Ju J., Lu G., Xiao A., Cancer prevention by tea and tea polyphenols. Asia Pac. J. Clin. Nutr. (2008) 17(S1), 245-248.

http://apjcn.nhri.org.tw/server/APJCN/17\%20Suppl\%201//245.pdf 
6. Intra J., Kuo S.M., Physiological levels of tea catechins increase cellular lipid antioxidant activity of vitamin $\mathrm{C}$ and vitamin $\mathrm{E}$ in human intestinal caco-2 cells.Chem. Biol. Interact. (2007),169, 91-99. https://doi.org/10.1016/j.cbi.2007.05.007

7. Sułkowska A., Maciazek-Jurczyk, M., Bojko B., Równicka J., Zubik-Skupien I., Temba E., Pentak D., Sułkowski W.W. Competitive binding of phenylbutazone and colchicines to serum albumin in multidrug therapy: A spectroscopic study. Journal of Molecular Structure. (2008) 881, 97-106.http://dx.doi.org/10.25177/JFST.2.1.1

8. Chaves O. A., Jesus C. S. H., Henriques E. S., Brito R. M. M., Serpa C. In-situ ultra-fast heat deposition does not perturb the structure of serum albumin. Photochemical \& Photobiological Sciences. (2016) 15, 1524-1535. https://pubs.rsc.org/en/content/articlelanding/2016/PP/c6pp00209a\#!divAbstract

9. Chaves O. A., Amorim A. P. O., Castro L. H. E., Sant’ Anna C. M. R., de Oliveira M. C. C., Cesarin-Sobrinho D., Netto-Ferreira J. C., Ferreira A. B. B. Fluorescence and docking studies of the interaction between human serum albumin and pheophytin. Molecules. (2015) 20, 1952619539. https://doi.org/10.3390/molecules201019526

10. Chaves O. A., da Silva V. A., Sant'Anna C. M. R., Ferreira A. B. B., Ribeiro T. A. N., de Carvalho M. G., Cesarin-Sobrinho D., Netto-Ferreira J. C., Binding studies of lophirone B with bovine serum albumin (BSA): Combination of spectroscopic and molecular docking techniques. Journal of Molecular Structure. (2017) 1128, pp. 606-611. https://doi.org/10.1016/j.molstruc.2016.09.036

11. Hu Y. J., Ou-Yang Y., Zhang Y., Liu Y. Affinity and specificity of ciprofloxacin-bovine serum albumin interactions: Spectroscopic approach. Protein J. (2010) 29, pp. 234-241. https://link.springer.com/article/10.1007\%2Fs10930-010-9244-6

12. Burmudzija A., Ratkovic Z., Muskinja J., Jankovic N., Rankovic B., Kosanic M., Đorđevic S. Ferrocenyl based pyrazoline derivatives with vanillic core: synthesis and investigation of their biological properties. RSC Adv. (2016) 6, 91420-91430. https://pubs.rsc.org/en/content/articlelanding/2016/ra/c6ra18977f/unauth\#!divAbstract

13. Amarowicz, R., Shahidi, F., Wiczkowski, W., Separation of individual catechins from green tea using silica gel column chromatography and HPLC. Journal of Food Lipids, (2003) 10(2), pp. 165-177. https://doi.org/10.1111/j.1745-4522.2003.tb00013.x

14. Larry D. Ward, Measurement of ligand binding to proteins by fluorescence spectroscopy, Methods in Enzymology, (1985) 117, pp. 400-414. https://doi.org/10.1016/S00766879(85)17024-2

15. Min Li, Ann E. Hagerman, Role of the Flavan-3-ol and Galloyl Moieties in the Interaction of (-)-Epigallocatechin Gallate with Serum Albumin. J. Agric. Food Chem. (2014) 62, pp. 3768-3775. https://pubs.acs.org/doi/pdf/10.1021/jf500246m

16. B. P. Kamat, J. Seetharamappa, M. B. Melwanki, Spectroscopic studies on the interaction of riboflavin with bovine serum albumin. Indian Journal of Biochemistry and Biophysics, (2004), 4, pp. 173-178. https://pdfs.semanticscholar.org/6920/07ad83210a1d0ac265502ac9038bc18bfed6.pdf

17. Morris G. M., Huey R., Lindstrom W., Sanner M. F., Belew R. K., Goodsell D. S., Olson A. J., Autodock4 and autodocktools4: automated docking with selective receptor flexibility. J. Comput. Chem. 2009, 30, pp. 2785-2791. https://doi.org/10.1002/jcc.21256

18. J. R. Lakowicz, Principles of Fluorescence Spectroscopy, Kluwer Academic/ Plenum Press, New York, 1999 (Chapters 8 and 13). http://nathan.instras.com/MyDocsDB/doc-800.pdf

19. DeLano W. L., The PyMol molecular graphics system. San Carlos, CA: DeLano Scientific (2004). http://www.pymol.sourceforge.net.

20. Sandip Pal, Chabita Saha, Maidul Hossain, Subrata Kumar Dey, Gopinatha Suresh Kumar, Influence of Galloyl Moiety in Interaction of Epicatechin with Bovine Serum Albumin: A Spectroscopic and Thermodynamic Characterization. PLoS One. (2012) 7(8), e43321. 
https://journals.plos.org/plosone/article?id=10.1371/journal.pone.0043321

21. Kagaya, N.; Kamiyoshi, A.; Tagawa, Y.; Nagashima, H.; Kawase, M.; Yagi, K. Interaction of rubratoxin B with serum albumin. Mycotoxins (2005) 55, pp. 23-26.

https://doi.org/10.2520/myco.55.23

22. Pettersen E. F., Goddard T. D., Huang C. C., Couch G. S., Greenblatt D. M., Meng E. C., et al. UCSF Chimera - a visualization system for exploratory research and analysis. J Comput Chem. (2004) 25(13), pp. 1605-1612. https://doi.org/10.1002/jcc.20084 